\title{
Programming settings and recharge interval in a prospective study of a rechargeable sacral neuromodulation system for the treatment of overactive bladder
}

\author{
Bertil Blok $^{1}$ (iD) | Philip Van Kerrebroeck ${ }^{2}$ | Stefan de Wachter ${ }^{3}$ | Alain Ruffion ${ }^{4}$ | \\ Frank Van der $\mathbf{A a}^{5} \mid$ Ranjana Jairam $^{2}$ (D) $\mid$ Marie Perrouin-Verbe $^{6} \mid$ Sohier Elneil $^{7}$
}

${ }^{1}$ Department of Urology, Erasmus M, edical Center, Rotterdam, The Netherlands

2 Department of Urology, Maastricht University Medical Centre, Maastricht, The Netherlands

${ }^{3}$ Department of Urology, University Hospital Antwerpen, Edegem, Belgium

${ }^{4}$ Department of Urology, Hôpital Lyon Sud, Pierre Bénite, Lyon, France

5 Department of Urology, UZ Leuven, Leuven, Belgium

${ }^{6}$ Department of Urology, University Hospital of Nantes, Nantes, France

${ }^{7}$ Department of Uro-Neurology, National Hospital of Neurology and Neurosurgery, London, United Kingdom

\section{Correspondence}

Bertil Blok, MD, PhD, Department of Urology, Erasmus Medical Center,

Rotterdam, The Netherlands.

Email: b.blok@erasmusmc.nl

Funding information

Axonics Modulation Technologies, Inc.
Aims: The RELAX-OAB study is designed to confirm the safety, efficacy, and technical performance of the Axonics r-SNM System, a miniaturized, rechargeable SNM system approved in Europe and Canada for the treatment of bladder and bowel dysfunction. The purpose of this article is to describe study subjects' ability to charge the rechargeable neurostimulator and to document their neurostimulator program settings and recharge interval over time.

Methods: Fifty-one OAB patients were implanted in a single-stage procedure. These results represent the 3-month charging experience for 48 subjects who completed the 3-month follow-up. Recharge intervals were estimated using therapy stimulation settings and subject experience was evaluated using questionnaires.

Results: Forty-seven of forty-eight (98\%) subjects were able to successfully charge their device prior to follow-up within 1-month post-implant. At 3-month post-implant, $98 \%$ of subjects were able to charge prior to their follow-up visit. Average stimulation amplitude across all subjects was $1.8 \mathrm{~mA}( \pm 1.1 \mathrm{~mA})$. A total of $69 \%$ of subjects had $\geq 14$-day recharge intervals (time between charging) and $98 \%$ of subjects had $\geq 7$-day recharge interval. No charging related adverse events occurred.

Conclusions: Study subjects were able to charge the Axonics r-SNM System and stimulation settings provided 2 weeks of therapy between recharging for most subjects. Subject satisfaction indicates that subjects are satisfied with rechargeable SNM therapy.

\section{K E Y W O R D S}

overactive bladder, patient experience, rechargeable, recharge interval, sacral neuromodulation, stimulation settings

Dr. Alan Wein led the peer-review process as the Associate Editor responsible for the paper.

This is an open access article under the terms of the Creative Commons Attribution-NonCommercial License, which permits use, distribution and reproduction in any medium, provided the original work is properly cited and is not used for commercial purposes. 


\section{1 | INTRODUCTION}

Over 250000 patients with bladder and bowel dysfunction have been treated with sacral neuromodulation (SNM) over the past 20 years. ${ }^{1}$ For these patients, the only commercialized SNM treatment option was a non-rechargeable SNM system that is replaced every 3-5 years when the neurostimulator battery depletes. System replacement procedures are burdensome to patients and physicians and are associated with surgical risk to the patient, including risk of infection and loss of efficacy. SNM practices and technology have evolved over time, most notably with the introduction of the tined lead and percutaneous implant procedure in the early 2000s. Studies have shown that the improved technology and techniques have increased therapy efficacy and decreased surgical revision and explant rates. ${ }^{2-4}$

Despite the improvements in some aspects of SNM, the therapy has lacked the technological innovation seen in other areas of neuromodulation, namely spinal cord stimulation (SCS) and deep brain stimulation (DBS). Rechargeable neuromodulation systems lasting 10 or more years in the body were introduced for SCS in $2006,{ }^{5}$ but were not available for SNM until recently. In 2016, the Axonics r-SNM System ${ }^{\mathrm{TM}}$, a miniaturized, rechargeable SNM system, obtained regulatory approval in Europe and Canada. The system is designed and tested to deliver therapy in the body for a minimum of 15 years and includes an implantable neurostimulator that is $5 \mathrm{cc}$ in volume, a $60 \%$ reduction in size compared to the $14 \mathrm{cc}$ Interstim II (Medtronic, Minneapolis, MN). An independent, expert review provides additional detail about SNM therapy and the characteristics and specifications of the Axonics r-SNM System. ${ }^{6}$

The RELAX-OAB study is a prospective, multi-center post-market clinical follow-up study designed with the primary aim of confirming the safety and technical performance of the Axonics r-SNM System. The aim of this study is to describe the usability and subject satisfaction with the rechargeable neurostimulator and document the study subjects' neurostimulator program settings and recharge interval based on the first 3 months of the RELAX-OAB study.

\section{2 | MATERIALS AND METHODS}

\section{1 $\mid$ Study design}

The RELAX-OAB study is a prospective, multi-center, single arm, open-label study with each subject serving as their own control. The study is being conducted according to the stipulations of the Declaration of Helsinki, ISO 14155: 2011 and applicable national regulations. The study protocol was approved by Ethics Committees at all study sites, and all subjects gave informed consent prior to participating in the study. Subjects were eligible for treatment with the Axonics r-SNM System if they met all inclusion and exclusion criteria. Additional study details were previously reported. ${ }^{7}$

\section{2 | Rechargeable SNM system}

The Axonics r-SNM System (Figure 1) is a miniaturized, implantable rechargeable SNM (r-SNM) system designed and tested to deliver therapy in the body for at least 15 years.

The Axonics r-SNM System includes an implantable neurostimulator that is $5 \mathrm{cc}$ in volume, which is over $60 \%$ smaller than the $14 \mathrm{cc}$ Interstim II. The neurostimulator connects to a 4-contact tined lead that is implanted through the sacral foramen using the same procedure previously described for other SNM systems. ${ }^{8}$ The system delivers constant current stimulation and provides adjustable stimulation parameters appropriate for SNM therapy. Implanted patients recharge their neurostimulator, expected to be needed once every 2 weeks, using an external charging unit placed over the implant. Stimulation parameters are programmed with a clinician programmer and stimulation intensity is controlled by the patient using a wireless remote control. An independent, expert review provides additional detail about SNM therapy and the characteristics and specifications of the Axonics r-SNM System. ${ }^{6}$

\section{3 | Follow-up visits}

Subjects were seen for follow-up visits at various time points up to 1-year post-implant, including 2-week and 1-month visits to assess initial response to therapy. Unscheduled follow-up visits were allowed as needed to adjust stimulation settings to optimize therapy. Data were collected on stimulation therapy settings at each visit, and outcomes were evaluated using a consecutive 3-day voiding diary, a symptom-related quality of life (QOL) surveys (ICIQOABqol, ICIQ-UI Short Form) and patient and physician satisfaction questionnaires. Therapy Responders were defined as subjects with a $\geq 50 \%$ improvement in their leaks or voids, or a reduction to $<8$ voids per day, on their 3-day diary at follow-up compared to baseline.

\section{4 | Recharge interval}

Estimates of the neurostimulator battery recharge interval were provided by Axonics Modulation Technologies, Inc., the manufacturer of the Axonics r-SNM System. Recharge interval estimates were based on active stimulation settings when patients completed their 3-month visits, including the stimulation amplitude, frequency, pulse width, and impedance. The estimates of the recharge interval assume the battery has been used for 15 years by utilizing factors that 

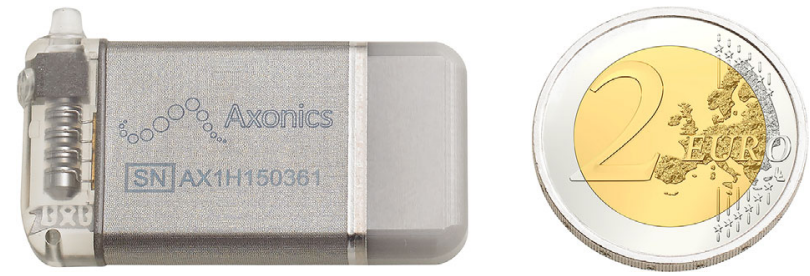

FIGURE 1 The Axonics r-SNM System includes a rechargeable, miniaturized neurostimulator that is $5 \mathrm{cc}$ in volume and has a 15 -year approved life in Europe and Canada

account for the effects on the battery capacity of aging and repeated discharging and recharging equivalent to 15 years of usage.

\section{5 | Adverse events}

All adverse events (AEs) were tracked and analyzed to assess the safety of the Axonics r-SNM System. A Data Safety Monitoring Board comprised of three expert clinicians not participating as investigators in the study, reviewed and adjudicated all adverse events.

\section{3 | RESULTS}

\section{1 | Subject overview}

Fifty-one subjects with $\mathrm{OAB}$ were implanted, and 48 subjects had data available at 3-month post-implant. One patient was explanted due to procedure-related infection and two subjects were lost to follow-up. Seventy-five percent of subjects were female and the average subject age was $51 \pm 15$ years (range: 21-77). Additional baseline subject characteristics, as well as the efficacy and safety outcomes of this study, were reported previously. ${ }^{7}$

\section{2 | Stimulation settings}

At implant and at 3 months all 48 subjects were programmed to bipolar stimulation. Table 1 shows the average stimulation settings across the subjects at each follow-up visit. Stimulation amplitude increased from $1.1 \pm 0.8 \mathrm{~mA}$ at implant to
$1.7 \pm 1.1 \mathrm{~mA}$ at 3 months, a $62 \%$ increase, however, most of the increase occurred by 2 weeks post-implant at which point the average amplitude was $1.5 \pm 1.1 \mathrm{~mA}$. Stimulation frequency was $14 \mathrm{~Hz}$ for all subjects at implant and at 3 months the average frequency was $14.3 \pm 1.6 \mathrm{~Hz}$ showing minimal change in stimulation frequency. Pulse width increased nominally from $209.4 \pm 4.3 \mu \mathrm{s}$ at implant to $210.6 \pm 11.6 \mu \mathrm{s}$ at 3 months. Impedance values decreased slightly from implant $(1005 \pm 292 \Omega)$ to 2 weeks $(946 \pm 187 \Omega)$ and then increased $27 \%$ on average to $1201 \pm 214 \Omega$ at 3 months. Cycling stimulation was not used for any subject.

Stimulation amplitude for the constant current rechargeable neurostimulator is programmed based on the current output (in $\mathrm{mA}$ ). Stimulation amplitudes at 3 months ranged from 0.4 to $5.8 \mathrm{~mA}$. A total of 30 of 48 subjects (63\%) had stimulation amplitudes less than $2 \mathrm{~mA}$ and $90 \%$ had amplitudes less than $3 \mathrm{~mA}$ (Figure 2).

\subsection{Estimated recharge interval}

Stimulation settings were used to estimate the recharge interval for the rechargeable neurostimulator. The method to determine the recharge interval was developed and provided by the manufacturer (Axonics Modulation Technologies, Inc., Irvine, CA). According to the manufacturer the estimated recharge interval is based on extensive device performance testing and represents the expected recharge interval after 15 years of use.

Almost all subjects (98\%) had at least a 7-day estimated recharge interval, and $69 \%$ of subjects had estimated recharge intervals $>14$ days (Figure 2). One subject had a 5-day recharge interval with stimulation settings of $5.6 \mathrm{~mA}$ (or $7.6 \mathrm{~V}), 14 \mathrm{~Hz}$ frequency, and $210 \mu$ s pulse width.

\section{4 | Ability to charge}

At 3 months $98 \%$ of subjects charged their neurostimulator without issue prior to their follow-up visit. A total of $96 \%$ of subjects successfully charged as early as within 2 weeks of implant. Subjects that were unable to charge at a follow-up visit were retrained and all were able to charge on their own prior to the next visit.

TABLE 1 Stimulation settings at follow-up visits

\begin{tabular}{llllll} 
& & \multicolumn{2}{c}{ Stimulation Settings $($ mean \pm std dev) } \\
\cline { 2 - 6 } Visit & $\boldsymbol{n}$ & Amplitude $(\mathbf{m A})$ & Frequency $(\mathbf{H z})$ & Pulse width $(\boldsymbol{\mu s})$ & Impedance $($ Ohms) \\
Post-implant & 51 & $1.1 \pm 0.8$ & $14 \pm 0$ & $209.4 \pm 4.2$ & $1005 \pm 292$ \\
2 weeks & 51 & $1.5 \pm 1.1$ & $14 \pm 0$ & $209.4 \pm 4.2$ & $946 \pm 187$ \\
1 months & 50 & $1.6 \pm 1.1$ & $13.9 \pm 0.6$ & $208.8 \pm 6$ & $964 \pm 161$ \\
3 months & 48 & $1.7 \pm 1.1$ & $14.3 \pm 1.6$ & $210.6 \pm 11.6$ & $1201 \pm 214$ \\
\hline
\end{tabular}




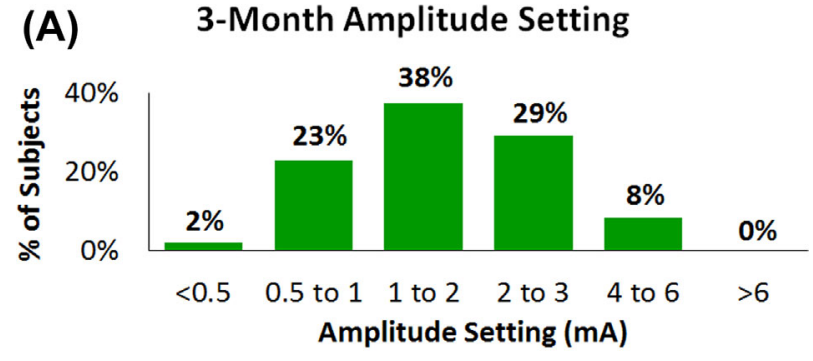

(B) Recharge Interval at 3-Month

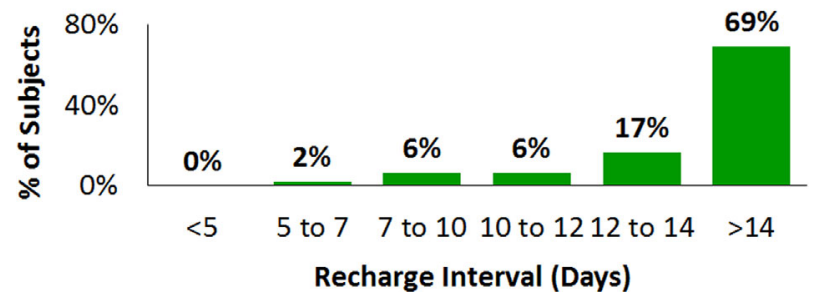

FIGURE 2 Therapy amplitude setting and estimated neurostimulator recharge interval at 3-month follow-up. A, Distribution of amplitude settings for all implanted subjects based on active stimulation settings when the patient departed the 3-month visit $(n=48)$. Amplitude setting is programmed using the Clinician Programmer. B, Distribution of estimated recharge interval for all implanted subjects based on stimulation settings at 3-month visit $(n=48)$. Estimation provided by Axonics Modulation Technologies, Inc

\subsection{Patient satisfaction}

The majority of subjects reported satisfaction with their SNM therapy at 3 months. Overall, $83 \%$ of implanted subjects were satisfied with their therapy, and when analyzed for only Therapy Responders the satisfaction rate rose to $94 \%$ (Figure 3). Most Therapy Responders, 76\%, were "Very Satisfied" with r-SNM therapy. Despite not meeting the clinical criteria for therapy success, $39 \%$ of Non-Responders were still "Very Satisfied" with their therapy. Additionally, $100 \%$ of subjects that responded to therapy reported they were at least "Likely" to recommend r-SNM therapy to a friend, and $88 \%$ said they would "Definitely" recommend the therapy to a friend.

\subsection{Charging-related adverse events}

Across all 51 implanted patients, no adverse events were reported related to the recharging of the neurostimulator.

\section{4 | DISCUSSION}

Sacral neuromodulation has been proven to reduce symptoms of bladder and bowel dysfunction and to improve patient quality of life. ${ }^{2-4}$ New rechargeable SNM systems may provide the ability to program patients, including utilizing higher power settings, without the need to maximize battery life associated with non-rechargeable SNM systems, which can require sacrificing efficacy to extend battery life or increasing programming visits to try advanced programming options (eg, cycling stimulation). This study provides evidence that subjects, particularly those experiencing therapeutic benefit, are satisfied with rechargeable sacral neuromodulation and recharging is neither a barrier for use nor an unwanted burden.

These results provide the first report of stimulation settings for subjects treated with a current-controlled, rechargeable sacral neuromodulation system. Most of the stimulation settings at 3-month post-implant were similar to those reported for the InSite trial, a 272-patient prospective multicenter study using a voltage controlled system (Interstim II). ${ }^{9}$ Stimulation frequency, pulse width, amplitude, and the associated impedance values were relatively consistent over this 3-month follow-up period. Change in stimulation accounted for the majority of any change in stimulation parameters and most of this occurred during the first 2 weeks in what would be considered an accommodation period. These findings suggest that the programming approach and preferred parameters are generally unchanged when programming this current controlled rechargeable system compared to the established practice for programming the voltage-controlled non-rechargeable system. One major difference, however, is that for this rechargeable system no patients had the cycling feature turned on while $35 \%$ of subjects in the InSite study had the cycling feature turned on at 3 months. Several studies have investigated the use of cycling as a means to extend the battery life of a non-rechargeable SNM system. ${ }^{10,11}$ However, a rechargeable system may offer a simplified programming process by reducing the use of cycling to extend battery life. Further, a rechargeable system may reduce the trade-offs made during programming when trying to optimize both efficacy and battery life. One subject in this study had a recharge interval $<7$ days due to high stimulation amplitude $(5.6 \mathrm{~mA}$ or $7.6 \mathrm{~V}$ ), and this subject could potentially be reprogrammed in the future with the cycling feature turned on to extend their recharge interval.

Several studies have reported high rates of patient satisfaction with rechargeable neuromodulation systems, ${ }^{12-15}$ including a strong preference for rechargeable systems in patients that previously had non-rechargeable system. ${ }^{12-14}$ However, this is the first report describing patient experience with a rechargeable SNM system. Patient preference and the appropriateness of a rechargeable system versus a nonrechargeable system may depend on a number of factors. A larger cohort is necessary to determine if there are predictive factors for whether a patient is better suited for a rechargeable or a non-rechargeable SNM system. A study examined which factors could predict whether a non-rechargeable or rechargeable system was more appropriate for patients receiving a SCS system for treatment of chronic pain. ${ }^{15}$ In this study of subjects with rechargeable SCS therapy, $20 \%$ of subjects reported that 


\section{(A) How satisfied are you with your r-SNM therapy?}

Responders

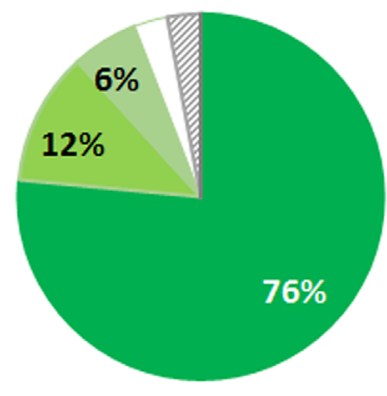

Non-Responders

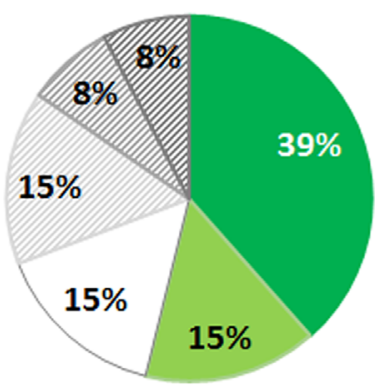

(B) How likely are you to recommend r-SNM therapy to a friend?

Responders

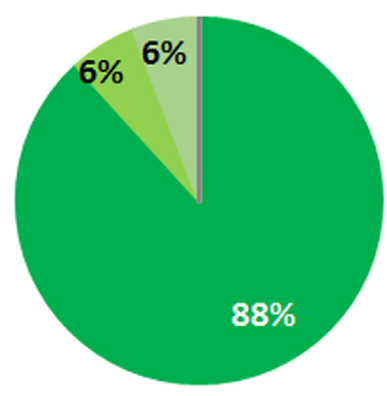

Very satisfied

Moderately satisfied

Slightly satisfied

$\square$ Neutral

Slightly dissatisfied

Moderately dissatisfied

Q Very dissatisfied
Non-Responders

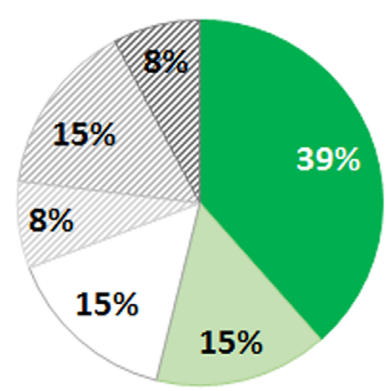

FIGURE 3 Subject satisfaction with r-SNM therapy at 3 months. A, Subject response at 3 months "How satisfied are you with your r-SNM therapy? (left) Satisfaction for therapy responders—subjects with $\geq 50 \%$ improvement in leaks and/or voids or a return to $<8$ voids per day ( $n=34$ ). (right) Satisfaction for therapy non-responders $(n=13)$. B, Subject response at 3 months to the question "How likely are you to recommend r-SNM therapy to a friend? (left) Likelihood response for therapy responders $(n=34)$. (right) Likelihood response for therapy non-responders $(n=13)$

recharging was not worth the added battery life of a 10-year rechargeable battery. These patients, compared to subjects reporting charging was worth the extra battery life, were significantly older (average of 74 vs 56 years), charged more frequently (average of 10 vs 5 times per month) and charged longer (2.4 vs $1.6 \mathrm{~h}$ per charge on average). ${ }^{15}$ However, selecting a rechargeable or non-rechargeable system for a patient based on recharge interval or duration is not practical given recharge interval and recharge duration are not known until the system is implanted and may change with reprogramming. Further, recharging 10 times per month for $2.4 \mathrm{~h}$ is not consistent with the charging experience of any subject in this study of rechargeable SNM. The rechargeable SNM system in this study has a more favorable neurostimulator battery life, with 15-year expected life and, based on this study, an estimated 2-4 recharges per month, suggesting a profile consistent with patients reporting recharging was a worthwhile tradeoff to get extended battery life.

Rechargeable neurostimulation systems have reported patient complaints related to excess heat generation during charging, ${ }^{15}$ however, no adverse events related to charging were reported in this study. The unique titanium-ceramic construction of the Axonics neurostimulator (Figure 1) is designed to provide safer recharging compared to rechargeable SCS devices. Additionally, the system has temperature sensors and software controls that pause charging if the patient's skin temperature increases significantly. These features suggest the rechargeable SNM system may not incur the charging related issues of SCS devices, and longer term follow-up of the subjects in this study will further evaluate these claims.

The limitation of this study is that specific questions regarding patient perceptions of recharging, including ease of use and burden relative to therapy benefit, were not included. Additionally, estimates of battery life are based on stimulation settings and an algorithm provided by the manufacturer. Future studies that provide patient reported or diary-based tracking of charging frequency and duration will provide valuable insights into the charging experience of patients using rechargeable sacral neuromodulation systems. 
The introduction of a rechargeable SNM system is expected to reduce the need for replacement surgeries due to battery depletion, resulting in fewer replacement surgeries and reduced lifetime costs of SNM therapy. The cost-benefit of rechargeable neuromodulation systems has been modeled for SNM systems, ${ }^{16}$ and the rechargeable device reduced was projected over a 15 -year period to reduce therapy costs to the United States healthcare system by $\$ 12$ Billion. ${ }^{16} \mathrm{~A}$ key aspect to realizing the cost benefit of rechargeable SNM therapy is the compliance of subjects with recharging their systems. The 3-month safety and efficacy results ${ }^{7}$ and high rates of therapy satisfaction in this report suggest that, in the short-term, subjects are not burdened by and are compliant with recharging their system. Long-term follow-up is necessary to confirm the benefits of rechargeable SNM therapy are maintained and to fully assess the cost-savings associated with rechargeable SNM therapy.

\section{5 | CONCLUSION}

The programming settings and estimated recharging intervals from this clinical study demonstrate that sacral neuromodulation with the Axonics r-SNM System is programmed at similar settings as non-rechargeable SNM and that patients are able to recharge and are not burdened by recharging their system. SNM has been shown to be a cost-effective option for third-line treatment of overactive bladder, and the cost-effectiveness and appeal of SNM therapy may be enhanced by the miniaturized, rechargeable SNM system.

\section{ACKNOWLEDGMENT}

Funding for the RELAX-OAB study was provided by Axonics Modulation Technologies, Inc.

\section{ORCID}

Bertil Blok (iD http://orcid.org/0000-0001-9354-7395

Ranjana Jairam (iD) http://orcid.org/0000-0001-9599-3284

\section{REFERENCES}

1. Medtronic press release. July 17, 2017. Five-year data demonstrate InterStim(TM) therapy offers sustained long-term efficacy and quality of life improvements for overactive bladder patients (newsroom.medtronic.com).

2. Siegel S, Noblett K, Mangel J, et al. Five year follow-up results of a prospective, multicenter study in overactive bladder subjects treated with sacral neuromodulation. J Urol. 2017. pii: S0022-5347(17) 77099-1. https://doi.org/10.1016/j.juro.2017.07.010

3. Groen J, Blok BF, Bosch JL. Sacral neuromodulation as treatment for refractory idiopathic urge urinary incontinence: 5-year results of a longitudinal study in 60 women. J Urol. 2011;186:954-959.
4. van Kerrebroeck PE, van Voskuilen AC, Heesakkers JP, et al. Results of sacral neuromodulation therapy for urinary voiding dysfunction: outcomes of a prospective, worldwide clinical study. J Urol. 2007;178:2029-2034.

5. Oakley JC, Krames ES, Prager JP, et al. A new spinal cord stimulation system effectively relieves chronic, intractable pain: a multicenter prospective clinical study. Neuromodulation. 2007;10: 262-278.

6. Cohn J, Kowalik C, Kaufman M, et al. Evaluation of the axonics modulation technologies sacral neuromodulation system for the treatment of urinary and fecal dysfunction. Expert Rev Med Devices. 2017;14:3-14.

7. Blok B, Van Kerrebroeck P, De Wachter S, et al. Three month clinical results with a rechargeable sacral neuromodulation system for the treatment of overactive bladder. Neurourol Urodyn. 2017;1-8. https://doi.org/10.1002/nau.23465

8. Spinelli M, Sievert K-D. Latest technologic and surgical developments in using InterStim ${ }^{\mathrm{TM}}$ therapy for sacral neuromodulation: impact on treatment success and safety. Eur Urol. 2008;54: 1287-1296.

9. Noblett KL, Mangel J, Bennett J, et al. Implantable neurostimulator programming at implant and follow-up in a large prospective trial of sacral neuromodulation therapy for overactive bladder patients. Female Pelvic Med Reconstr Surg. 2014;20:S367-S368.

10. Cadish LA, Osann KE, Noblett KL. Stimulation latency and comparison of cycling regimens in women using sacral neuromodulation. Neurourol Urodyn. 2017;36:486-489.

11. Hoen L, Groen J, Scheepe J, Blok BF. Intermittent sacral neuromodulation for idiopathic urgency urinary incontinence. Neurourol Urodyn. 2017;36:385-389.

12. McAuley J, Farah N, van Gröningen R, Green C. A questionnairebased study on patients' experiences with rechargeable implanted programmable generators for spinal cord stimulation to treat chronic lumbar spondylosis pain. Neuromodulation. 2013;16: 142-146.

13. Sciacca S, Smith JS, Akram H, Asim A, Matharu M, Watkins L. Rechargeable occipital nerve stimulator systems: a patient satisfaction study. Br J Neurosurg. 2014;28:645-649.

14. Waln O, Jimenez-Shahed J. Rechargeable DBS IPGs in movement disorders: patient satisfaction and conversion parameters. Neuromodulation. 2014;17:425-430.

15. Lam CK, Rosenow JM. Patient perspectives on the efficacy and ergonomics of rechargeable spinal cord stimulators. Neuromodulation. 2010;13:218-223.

16. Noblett K, Dmochowski R, Vasavada S, et al. Cost profiles and budget impact of rechargeable versus non-rechargeable sacral neuromodulation devices in the treatment of overactive bladder syndrome. Neurourol Urodyn. 2017;36:727-733.

How to cite this article: Blok B, Van Kerrebroeck P, de Wachter S, et al. Programming settings and recharge interval in a prospective study of a rechargeable sacral neuromodulation system for the treatment of overactive bladder. Neurourology and Urodynamics. 2018;37:S17-S22. https://doi.org/10.1002/nau.23476 